\title{
Research article \\ Comparison of serum nitric oxide levels with case severity in pre-eclampsia - A case control study
}

\section{Sangappa Virupaxappa Kashinakunti ${ }^{1,2}$, Basavaraj Devaranavadagi ${ }^{3}$, Manjula Rangappa ${ }^{4}$, Ashalata Mallapur ${ }^{5}$}

${ }^{1} \mathrm{PhD}$ Scholar, ${ }^{3}$ Professor and Head, Department of Biochemistry, Shri B M Patil Medical College, BLDE University, Vijayapur, Karnataka, India

${ }^{2}$ Professor, Department of Biochemistry, ${ }^{4}$ Professor, Department of Community Medicine, ${ }^{5}$ Professor and Head, Department of Obstetrics and Gynaecology, S. Nijalingappa Medical College, Navanagar, Bagalkot 587102, Karnataka, India

(Received: September 2020

Revised: February 2021

Accepted: March 2021)

Corresponding author: Sangappa V. Kashinakunti. Email: drsvkashinakunti@yahoo.co.in

\begin{abstract}
Introduction and Aim: In preeclampsia (PE) due to abnormal trophoblastic invasion of the maternal spiral arteries leads to decreased uteroplacental circulation. Nitric oxide (NO) is involved in regulation of feto-placental vascular permeability and resistance. It also affects maturation, development of the placenta and foetal survival. Maternal serum NO levels are controversial in PE and only few studies have focused on birth outcomes. Hence, the current study was undertaken to estimate and compare the maternal serum NO levels in normal pregnant and PE women and also see the correlation between maternal serum NO level with severity of PE and to study its association with birth outcome.
\end{abstract}

Materials and Methods: The study was conducted at a tertiary care hospital in North Karnataka, India. Forty singleton primigravida PE patients diagnosed and classified as mild/severe PE, as per ACOG guidelines and 40 healthy pregnant were selected for the study. NO was estimated by ELISA method.

Results: NO was high in PE cases than controls but it was not statistically significant. But NO was significantly $(\mathrm{p}=0.03)$ higher in mild PE than severe PE patients. The NO level negatively correlated significantly $(\mathrm{p}=0.031)$ with case severity. Maternal serum NO did not show any significant correlation with birth weight and APGAR score of the new born baby.

Conclusion: In mild PE rise in maternal serum NO, may be to due compensatory mechanism to normalise persistently elevated blood pressure and decreased level of serum NO in severe PE may be due to down-regulation of the nitric oxide synthase.

Keywords: Nitric oxide; pre-eclampsia; birth weight; APGAR score.

\section{INTRODUCTION}

$\mathrm{P}$ reeclampsia $(\mathrm{PE})$ is a hypertensive multisystem disorder in previously normotensive women, complicating $6-10 \%$ of all pregnancies over 20 weeks of gestation. World Health Organisation estimates that frequency of PE is seven times higher in developing countries than developed countries. PE is one of the leading causes of foetal growth disorders, foetal morbidity and mortality. There is a five-fold increase in perinatal deaths from intrauterine growth restriction and prematurity as a result of PE. Maternal complications of PE include eclampsia, stroke, liver and renal failure, disseminated intravascular coagulation, premature labour and maternal death (1-4).

Evidence suggests that general vascular endothelial dysfunction, which occurs during PE can explain mechanism responsible for its pathogenesis. Primary disturbance appears to result from reduced uteroplacental circulation due to abnormal trophoblastic invasion of the maternal spiral arteries resulting in poor placentation. These spiral arteries retain their endothelial lining and the underlying smooth muscles, which make them reactive to vasoactive agents expressed by the endothelial cells. Endothelial cell dysfunction can cause hypertension with its increased production of vasoconstrictor agents such as plasma endothelin or reduced release of vasodilator agents such as Nitric oxide (NO) and prostacyclin (5-7).

NO is a physiological agent involved in regulation of feto-placental vascular permeability and resistance and platelet aggregation in the placenta. Maturation and development of the placenta and foetal survival is significantly affected by nitric oxide. Endothelial nitric oxide produces its action through cyclic guanosine monophosphate dependent pathway. Serum NO level is considered as a valuable biomarker of endothelial function. NO biosynthesis data are also controversial in PE (7-9).

The outcome of current study may give more sight to clinicians as to whether the pathogenesis of $\mathrm{PE}$ is linked to maternal serum NO levels and how such knowledge can be utilized in effective prevention of PE. Some authors depicted different results and they showed that the biosynthesis of NO in preeclampsia was decreased, unchanged or raised and hence, this issue is still debatable (10-12). Hence the current 
study was undertaken to estimate and compare maternal serum levels of NO in normal pregnant and PE women; and also to find the correlation between serum nitric oxide level with severity of $\mathrm{PE}$ and to study its association of NO levels with birth outcome.

\section{MATERIALS AND METHODS}

This is a case control study, conducted in department of obstetrics and gynecology and department of biochemistry at Hanagal Shri Kumareshwara Hospital, Bagalkot, Karnataka India, from Jan 2019 to Jun 2019. The study was approved by Institutional ethics committee. Informed consent was obtained from all the participants.

Primigravida (singleton) pregnant women aged 18-35 years, 20 or more weeks of gestation, diagnosed as $\mathrm{PE}$ and classified as mild/severe PE based on America college of obstetricians and gynecologists guidelines (13) were selected for the study. Patients with pre-mature rupture of membranes, chorioamnionitis, multiple gestation, Rh isoimmunization, fetal anomalies, intra uterine fetal death, chronic inflammatory diseases, history of diabetes mellitus, past history of systemic hypertension, cardiovascular or renal diseases and chronic inflammatory diseases were excluded from the study. Healthy pregnant women, matched for age, gravida and gestational weeks with the cases were selected as controls.

All participants were subjected for history taking and followed by general physical examination.
Abdominal examination was performed for fundal level, lie and presentation of fetus. Blood samples were collected by venipuncture in two tubes, $2 \mathrm{ml}$ with anticoagulant and $2 \mathrm{ml}$ without anticoagulant. Complete blood count, blood grouping and rhesus factor were done. After clotting, the samples were centrifuged and serum was pipetted and the following parameters were measured blood glucose, liver function tests, urea. serum creatinine, uric acid and nitric oxide. Sample for NO measurement was stored at $-20^{\circ} \mathrm{C}$ till estimation. Urine analysis for proteinuria was done. Abdominal ultrasonography was done for gestational age, fetal heart sound. All the participants were visited again at the outcome of the pregnancy and the outcome findings was noted.

Sample size calculation was done using Open Epi software version $2.3: 1$, retrospectively with $83.47 \%$ power of the study, using case severity in to consideration, sample size calculated was 34 40. Hence, 40 preeclampsia cases and 40 normal gestation controls were included in the study.

Analysis was done using SPSS software version 19. Unpaired ' $t$ ' test for quantitative data and Pearson's correlation tests were applied. $\mathrm{P}<0.05$ will be considered as statistically significant.

\section{RESULTS}

Seven women were mild PE and 33 were severe PE patients.

Table 1: Diagnostic and severity features of healthy pregnant women and preeclampsia group

\begin{tabular}{|c|c|c|c|c|}
\hline & Controls & Cases & t & p \\
\hline Gestational weeks & $37.78 \pm 2.97$ & $35.63 \pm 4.37$ & 2.511 & 0.014 \\
\hline Pulse Beats/min & $86.54 \pm 6.98$ & $92.28 \pm 9.27$ & -3.044 & 0.003 \\
\hline SBP mmHg & $117.41 \pm 10.75$ & $157.55 \pm 17.75$ & -11.878 & 0.000 \\
\hline DBP mmHg & $74.70 \pm 9.45$ & $102.40 \pm 9.10$ & -13.093 & 0.000 \\
\hline BMI & $24.82 \pm 3.65$ & $28.99 \pm 5.41$ & -3.008 & 0.004 \\
\hline Haemoglobin g\% & $11.57 \pm 1.48$ & $11.06 \pm 2.12$ & 1.19 & 0.23 \\
\hline Platelet & $228891.89 \pm 49402.42$ & $199743.59 \pm 81379.78$ & -0.67 & 0.50 \\
\hline RBG mg/dl & $88.52 \pm 21.46$ & $85.87 \pm 20.37$ & 0.563 & 0.575 \\
\hline Serum Urea mg/dl & $21.30 \pm 6.48$ & $24.28 \pm 4.91$ & -2.291 & 0.025 \\
\hline Serum Creatinine mg/dl & $0.73 \pm 0.13$ & $0.75 \pm 0.15$ & -0.542 & 0.590 \\
\hline AST IU/L & $23.37 \pm 10.40$ & $43.43 \pm 61.28$ & -2.015 & 0.04 \\
\hline ALT IU/L & $15.82 \pm 10.17$ & $35.36 \pm 67.37$ & -1.791 & 0.07 \\
\hline Urine Protein mg/l & $120.26 \pm 158.84$ & $2464.68 \pm 3185.03$ & -2.73 & 0.010 \\
\hline
\end{tabular}

SBP - Systolic blood pressure; DBP- Diastolic blood pressure; BMI- Body mass index; RBG- Random blood glucose; Urine P/C ratio- Urine protein creatinine ratio; AST- Aspartate transaminase; ALT- Alanine transaminase.

There was significant decrease in gestational weeks in preeclampsia. Pulse, systolic blood pressure, diastolic blood pressure and body mass index were significantly higher in preeclampsia women than normal pregnant women. Haemoglobin and Platelet count were less in preeclampsia cases than the control group but it was not statistically significant. All renal function tests were significantly higher in preeclampsia cases than healthy pregnant women except serum creatinine which also more was in cases than control group but it was not statistically significant. Aspartate transaminase was significantly higher in cases than the control group, alanine transaminase was also more in preeclampsia cases than normal pregnant women, but it was not statically significant. 
Table 2: Nitric oxide (NO) in controls and cases

\begin{tabular}{|c|c|c|c|c|}
\hline & Controls & Cases & t & p \\
\hline NO $\boldsymbol{\mu m o l} / \mathbf{l}$ & $76.38 \pm 117.02$ & $115.77 \pm 195.32$ & -1.090 & 0.279 \\
\hline
\end{tabular}

NO was also high in cases than controls, but it was not statistically significant.

Table 3: Nitric oxide (NO) in mild and severe cases of PE

\begin{tabular}{|c|c|c|c|c|}
\hline & Mild & Severe & t & p \\
\hline NO $\boldsymbol{\mu m o l} / \mathbf{l}$ & $165.27 \pm 217.77$ & $58.39 \pm 77.86$ & 2.24 & 0.03 \\
\hline
\end{tabular}

NO was significantly higher in mild PE patients than severe PE patients.

Table 4: Co-relation of Nitric oxide (NO) with case severity

\begin{tabular}{|l|c|c|}
\hline & Pearson Correlation & $\mathbf{p}$ \\
\hline NO & -0.350 & 0.031 \\
\hline
\end{tabular}

Significant negative correlation was between NO and case severity of PE, which meant as the severity of PE increases the NO levels, would decrease.

Table 5: Correlation between maternal serum Nitric oxide (NO) level with birth outcome

\begin{tabular}{|c|c|c|c|}
\hline & Birth weight & APGAR 1 min & APGAR 5 min \\
\hline r & -0.020 & 0.079 & 0.055 \\
\hline p & 0.909 & 0.663 & 0.762 \\
\hline
\end{tabular}

NO did not show any significant correlation with birth weight and APGAR score at 1 minute and 5 minutes.

\section{DISCUSSION}

Many studies showed that the biosynthesis of NO was increased in normal pregnancy, especially in the second trimester, with its peak in the third trimester of pregnancy. Study done by Adu-Bonsaffoh et al., (14) found that PE women had a significantly higher maternal serum nitrite concentration $(p<0.001)$ while women with chronic hypertension had a significantly lower nitrite concentration than the control group $(\mathrm{P}=0.04)$. Women with gestational hypertension had a similar nitrite level to the control group (15-17).

In the current study, there was increase in maternal serum NO levels of PE women compared to normal healthy pregnant women, but it was not statistically significant $(\mathrm{p}=0.279)$. Literature has reported inconsistent results as far as serum nitric oxide levels in PE compared to healthy pregnant women.

They attributed the marked increase in NO levels to compensatory mechanism to the pathological effect of PE. It is possible that the amount of bioavailable NO needed to restore the persistently elevated BP was insufficient (14).

Conversely, Darkwa et al., (18) showed a statistically non-significant reduction in plasma NO levels in PE compared to healthy pregnant women $(\mathrm{P}=0.160)$ agreeing with the observations of other studies. The studies have indicated that maternal serum NO levels are reduced in PE. Sahu et al., (19) reported a reduction in NO production in PE women compared with normotensive pregnant women. The reduction in $\mathrm{NO}$ as a result of down-regulation of the nitric oxide synthase enzyme, leading to a relative deficiency of $\mathrm{NO}$ and an excess of ONOO- and/or occurrence of endothelial damage in the development of the disorder, the production of O2- by NOS is central to the pathophysiology eventually leading to clinical syndrome and NO donors have been hypothesized to prevent PE. Another reason for reduced NO serum levels of PE women could be due to increased binding and reduced releasing of nitrates from red blood cells $(4,20,21)$.

The determination of nitric oxide levels is confounded by several internal factors including a source of sample (plasma, serum, urine), external factors like method of assaying, diet rich in nitrates such as cured meats or vegetables, alcohol consumption, atmospheric pollution, exercise and cigarette smoking. It may be difficult to find a significant change over the uncontrolled external factors and eliminate the inter-subject variations (18, 23).

In the present study, the serum NO levels were compared between the mild and severe PE women, the results showed significantly higher level of $\mathrm{NO}$ in mild PE patients compared to severe PE women. There was a significant negative correlation between maternal serum NO and severity of PE.

However, a study of Adu-Bonsaffoh et al., (14) showed markedly elevated serum levels of NO in early-onset PE (Severe form) compared to late-onset $\mathrm{PE}$ that is considered as a mild disease. This may represent a compensatory mechanism to offset the ongoing pathological process and indicating the generalized endothelial dysfunction. On the other hand, other researchers have reported that circulating nitrate and nitrite levels are not reduced in patients with severe PE compared with normotensive controls $(11,14,24)$. 
Erdemoğlu et al., concluded that there was no difference between means of NO in healthy, mild, and severe preeclamptic patients, neither severity of preeclampsia nor eclampsia was correlated with NO values (24). Shaker et al., in their study showed that the, mean levels of serum NO products were significantly decreased in both the mild and severe groups compared with the normotensive group ( $\mathrm{p} 1$, $\mathrm{p} 2<0.001$ ), but no significant difference was found between the mild and severe groups (p3 > 0.05). They also did not get any correlation between maternal serum NO and birth weight. In the current study also there was no correlation between maternal serum NO and birth outcomes viz birth weight and APGAR score (25).

The limitation of present study was that the diet history was not considered and history of alcohol consumption and smoking were not included because these are culturally sensitive issues in this part of India. Further longitudinal nitric oxide synthase estimation and genetic expression are required to find the facts of pathogenesis of PE.

\section{CONCLUSION}

In conclusion, in mild PE, significant rise in maternal serum NO may be due the compensatory mechanism to normalise persistently elevated blood pressure where the regulatory mechanism works at its zenith; and decreased level of serum NO in severe PE may be due to down-regulation of the nitric oxide synthase, which ultimately leads to endothelial dysfunction, presenting as a syndrome.

\section{CONFLICT OF INTEREST}

Authors declare no conflicts of interest.

\section{REFERENCES}

1. Bernardi, F. C., Vuolo, F., Petronilho, F., Michels, M., Ritter, C., Dal-Pizzol, F. C. Plasma nitric oxide, endothelin1 , arginase and superoxide dismutase in the plasma and placentae from preeclamptic patients. An Acad Bras Cienc. 2015; 87(2): 713-719.

2. Carty, D. M., Delles, C., Dominiczak, A. F. Preeclampsia and future maternal health. J Hypertens 2010; 28: 13491355.

3. World Health Organization. Make every mother and child count. The world health report 2005. Geneva, Switzerland: World Health Organization, 2005. Available at: http://www.who.int/whr/2005/whr2005_en.pdf (Accessed on 30 June 2017)

4. Gilbert, J. S., Ryan, M. J., La Marca, B. B., Sedeek, M., Murphy, S. R., Granger, J. P. Pathophysiology of hypertension during preeclampsia: linking placental ischemia with endothelial dysfunction. Am J Physiol Heart Circ Physiol 2008; 294(2): 541-550.

5. George, E. M., Granger, J. P. Recent insights into the pathophysiology of preeclampsia. Expert Rev Obstet Gynecol 2012; 5(5): 557-556.

6. Su, J. B. Vascular endothelial dysfunction and pharmacological treatment. World J Cardiol 2015; 26: 719741.

7. Myatt, L. Placental adaptive responses and fetal programming. J Physiol 2006; 572: 25-30.
8. Krause, B. J., Hanson, M. A., Casanello, P. Role of nitric oxide in placental vascular development and function. Placenta 2011; 32: 797-805.

9. Suzuki, T., Mori, C., Yoshikawa, H., Miyazaki, Y., Kansaku, N., Tanaka, K., et al., Changes in nitric oxide production levels and expression of nitric oxide synthase isoforms in the rat uterus during pregnancy. Biosci Biotechnol Biochem. 2009; 73: 2163-2166.

10. Matsubara, K., Matsubara, Y., Hyodo, S., Katayama, T., Ito, M. Role of nitric oxide and reactive oxygen species in the pathogenesis of preeclampsia. J Obstet Gynaecol Res. 2010; 36: 239-247.

11. Zeng, Y., Li, M., Chen, Y., Wang, S. Homocysteine, endothelin-1 and nitric oxide in patients with hypertensive disorders complicating pregnancy. Int J Clin Exp Pathol. 2015; 8: 15275-15279.

12. Acauan Filho, B. J., Pinheiro da Costa, B. E., Ogando, P. B., Vieira, M. C., Antonello, I. C., Poli-de Figueiredo, C. E. Serum nitrate and NOx levels in preeclampsia are higher than in normal pregnancy. Hypertens Pregnancy. 2016; 35: 226-233.

13. American College of Obstetricians and Gynecologists; Task Force on Hypertension in Pregnancy. Hypertension in pregnancy. Report of the American College of Obstetricians and Gynecologists' Task Force on Hypertension in Pregnancy. Obstet Gynecol. 2013; 122: 1122-1131.

14. Adu-Bonsaffoh, K., Antwi, D. A., Obed, S. A., Gyann, B. Nitric oxide dysregulation in the pathogenesis of preeclampsia among Ghanaian women. Integrated Blood Pressure Control 2015; 8: 1-6.

15. Osol, G., Ko1, N. L., Mandalà, M. Altered Endothelial Nitric Oxide Signaling as a Paradigm for Maternal Vascular Maladaptation in Preeclampsia. Curr Hypertens Rep 2017; 19: 82.

16. Pimentel, A.M.L., Pereira, N. R., Costa, C. A., Mann, G. E., Cordeiro, V.S.C., de Moura, R. S., et al., L-arginine-nitric oxide pathway and oxidative stress in plasma and platelets of patients with pre-eclampsia. Hypertens Res. 2013; 36(9): 783-788.

17. Eleuterio, N. M., Palei, A.C.T., Machado, J.S.R., TanusSantos, J. E., Cavalli, R. C., Sandrim, V. C., et al. Relationship between adiponectin and nitrite in healthy and preeclampsia pregnancies. Clin Chim Acta. 2013; 423: 112-115.

18. Darkwa, E. O., Djagbletey, R., Essuman, R., Sottie, D., Dankwah, G. B., Aryee, G. Nitric Oxide and Pre-Eclampsia: A Comparative Study in Ghana. Open Access Maced J Med Sci. 2018 Jun 20; 6(6): 1023-1027.

19. Sahu, S., Daniel, M., Abraham, R., Vedavalli, R., Senthilvel, V. Study of uric acid and nitric oxide concentrations in preeclampsia and normal pregnancy. Int $\mathbf{J}$ Biol Med Res. 2011; 2(1): 390-393.

20. Diejomaoh, F., Omu, A., Al-Busiri, N., Taher, S., AlOthman, S., Fatinikun, T., et al., Nitric oxide production is not altered in preeclampsia. Arch Gynecol Obstet. 2004; 269(4): 237-243.

21. Poorolajal, J., Jenabi, E. The association between body mass index and preeclampsia: a meta-analysis. J Matern Neonatal Med 2016; 29(22): 3670-3676.

22. Owusu, B. Y., Stapley, R., Honavar, J., Patel, R. P. Effects of erythrocyte aging on nitric oxide and nitrite metabolism. Antioxid Redox Signal 2013; 19: 1198-1208.

23. Hodžić, J., Izetbegović, S., Muračević, B., Iriškić, R., Jović, H. S. Nitric oxide biosynthesis during normal pregnancy and pregnancy complicated by preeclampsia. Med Glas (Zenica). 2017; 14(2): 211-217.

24. Erdemoğlu, E., Uğur, M., Erdemoğlu, E. Plasma homocysteine and nitric oxide levels in preeclampsia. J Turkish-German Gynecol Assoc. 2009; 10: 26-29.

25. Shaker, O. G., Hany Shehata, H. Early Prediction of Preeclampsia in High-Risk Women. Journal of women's health $2011 ; 20(4)$ : 539-544. 